\title{
Correct Identification and Explanation of Age-Related Changes Are Always Possible
}

\author{
Peter C.M. Molenaar \\ University of Amsterdam, The Netherlands
}

\section{Key Words}

Age-related changes, identification - Research design • Longitudinal design

\begin{abstract}
In this commentary, it is argued that the definition of the time or the age variable in the models presented by Hofer and Sliwinski is ambiguous, and a standard interpretation of this time of age variable is proposed instead. Some simulation studies are presented, the results of which indicate that, even with age-heterogeneous crosssectional data, correct identification of the true sources of age-dependent variation is possible. An important qualification, based on new methodological work in progress, is given of the scope of longitudinal designs. It is concluded that all kinds of age-related changes are potentially interesting and can be correctly identified and explained.
\end{abstract}

Copyright $@ 2002$ S. Karger AG, Basel

\section{Introduction}

I have read with great interest the paper by Hofer and Sliwinski [1] (to which I will henceforth refer to as 'the authors'). It deals with some of the methodological issues when using cross-sectional designs in order to investigate age-dependent changes. In what follows I will present a critical discussion of various aspects of the paper. Analytic discourse necessarily has a critical undertone. Therefore, I would like to stress at the outset that the authors deserve praise because they make a fundamental statement and raise important questions about the information which can be obtained from age-heterogeneous crosssectional data.

This commentary is organized as follows. First I will shortly discuss the basic equation 1 given in the paper. From that discussion I will draw some conclusions concerning the correctness of the remaining equations. Second, I will present some results from simulation studies based on the basic equation 1 given in the paper. The results of these simulation studies indicate that, even with age-heterogeneous cross-sectional data, correct identification can be made of the true sources of age-dependent variation. I also will consider the remarks about partialling out of age made by the authors. Third, I will comment on the statements made by the authors about longitudinal analysis. These comments will include new methodological developments that qualify the type of information which can be obtained even from what is regarded as the most powerful design to study within-subject variation, namely the longitudinal design. In closing this commentary, some general remarks will be made concerning designs with several homogeneous age groups and the relation thereof with the age-homogeneous cross-sectional and sequential designs favored by the authors. I end with a general evaluation of the paper.

\begin{tabular}{ll}
\hline KARGER & ( ) 2002 S. Karger AG, Basel \\
Fax +4161306 1234 & 0304-324X/02/0481-0013\$18.50/0 \\
$\begin{array}{l}\text { E-Mail karger@karger.ch } \\
\text { www.karger.com }\end{array}$ & $\begin{array}{l}\text { Accessible online at: } \\
\text { www.karger.com/journals/ger }\end{array}$
\end{tabular}

Peter C.M. Molenaar

University of Amsterdam

Roetersstraat 15, Room 502

NL-1018 WB Amsterdam (The Netherlands)

E-Mail op_molenaar@macmail.psy.uva.nl 


\section{Modeling Issues}

Equation 1.1 in the paper, which for the convenience of the reader is repeated below, is the basic equation from which all other equations are derived under several additional assumptions concerning the model parameters.

$$
\mathrm{X}_{\mathrm{it}}=\mathrm{L}_{\mathrm{x}}+\mathrm{L}_{\mathrm{xi}}+[\mathrm{t}] \mathrm{S}_{\mathrm{x}}+[\mathrm{t}] \mathrm{S}_{\mathrm{xi}}+\mathrm{e}_{\mathrm{xi}}
$$

A similar equation is given for another variable $y_{i t}$. It is indicated by the authors that each variable with subscript $\mathrm{i}$ is a random variable. In the psychometrical literature it is common practice to refer to equation 1 as describing the $\mathrm{x}$-score of subject $\mathrm{i}$ randomly chosen from the population of interest, in short, random subject i [2].

My main question about equation 1 concerns the status of the variable [t], i.e., the age of subject $i$. It has no subscript i. Does that imply that it is not a random variable? In equations 3-5 the age of subject $i$ has subscript $i$ : $\left[\mathrm{t}_{\mathrm{i}}\right]$. Does this imply that the age is a random variable? In equation 6 the age variable again has no subscript $i$, suggesting that it is not a random variable. This inconsistent treatment of the age variable is not merely an academic issue. I will show that the correct and consistent treatment of the age variable has major consequences for covariance expressions derived from equation 1 .

Equation 1 describes the $x$-score of random subject $i$, i.e., it denotes the score of an arbitrary given subject drawn from some homogeneous population of mutually interchangeable subjects. This implies that the age of this subject $i$ also is a random variable. It is a random variable in the population of subjects, from which the given subject $i$ is drawn randomly. Random sampling from a population constitutes one of the two main interpretations of randomness in the psychometric literature. Ellis and Junker [2] give an excellent discussion of these interpretations and their relationships. According to this interpretation, the age variable should have subscript $i$ in all equations given in the paper, not only in equations 3-5.

Of course, age is a strange random variable in the population of subjects. In his masterly treatise about the study of behavioral development, Wohlwill [3] interpreted age as a kind of index variable and gave strong arguments not to use age as an explanatory variable. Yet this is exactly the role of the age variable in equation 1 : it is an explanatory variable for the age-dependent variation of the x-scores. Wohlwill [3] would have considered equation 1 ill-suited to the study of development. Notwithstanding this, however, even when interpreted as an index variable, age is a random variable in the population of subjects. And in case it is used in a model of development like equation 1 , it should be treated consistently as a random variable.

The ambiguity of the authors about the proper treatment of the age variable can perhaps most clearly be illustrated in a comparison of their equations 5 and 6 . The right-hand side of equation 5 equals $\mathrm{S}_{\mathrm{x}} \mathrm{S}_{\mathrm{y}} \operatorname{var}\left(\mathrm{t}_{\mathrm{i}}\right)$ which is the product of the fixed variables $S_{x}$ and $S_{y}$ and the variance of the age variable $\left[\mathrm{t}_{\mathrm{i}}\right]$. Hence in equation 5 , denoting cov $(\mathrm{X}, \mathrm{Y})$, age $\left[\mathrm{t}_{\mathrm{i}}\right]$ is considered to be a random variable. In contrast, in equation 6 , again denoting $\operatorname{cov}(\mathrm{X}, \mathrm{Y})$, the last component of the right-hand site equals $\left[\mathrm{t}^{2}\right] \operatorname{cov}\left(\mathrm{S}_{\mathrm{xi}}\right.$, $\mathrm{S}_{\mathrm{yi}}$ ). Hence this component denotes the product of the covariance between the random variables $S_{x i}$ and $S_{y i}$ and the square of the age variable $[t]$. Hence in equation 6 , age [t] is considered to be a fixed variable. This observation about the inconsistent treatment of the age variable is independent of the further details of the equations 5 and 6 , in particular whether the remaining variables (e.g., $S_{x}$ and $S_{x i}$ ) are random or not. Incidentally, the term $\left[\mathrm{t}^{2}\right]$ of the last component $\left[\mathrm{t}^{2}\right] \operatorname{cov}\left(\mathrm{S}_{\mathrm{xi}}, \mathrm{S}_{\mathrm{yi}}\right)$ of equation 6 is in my view meaningless, like the term $[\mathrm{t}]$ in the second and third components of the right-hand site of equation 6 . The (square of the) age of which individual is being denoted here? I expect that the authors mean by these terms: $[t]=$ mean age; $\left[\mathrm{t}^{2}\right]=$ mean sum of squares of ages.

If the age variable is consistently treated as a random variable in the population of subjects, it then follows that two equations in the paper are not correct. The reason for this is the occurrence of a product of random variables in equation 1 , namely $\left[\mathrm{t}_{\mathrm{j}}\right] \mathrm{S}_{\mathrm{xi}}$. The variance $\operatorname{var}\left(\left[\mathrm{t}_{\mathrm{j}}\right] \mathrm{S}_{\mathrm{xi}}\right)$ and the covariance $\operatorname{cov}\left(\left[\mathrm{t}_{\mathrm{i}}\right] \mathrm{S}_{\mathrm{xi}},\left[\mathrm{t}_{\mathrm{i}}\right] \mathrm{S}_{\mathrm{yi}}\right)$ now become fourth-order moments involving the random variables $\left[\mathrm{t}_{\mathrm{i}}\right], \mathrm{S}_{\mathrm{xi}}$, and $\mathrm{S}_{\mathrm{yi}}$. This implies that equation 6 is incorrect, because it should include such fourth-order moments under a consistent treatment of age as a random variable. The required expressions under the assumption of normal distributions for the random variables concerned can be found in, e.g., Molenaar et al. [4]. Suddenly treating age as a fixed variable in equation 6 would seem to alleviate this problem, but only at the unacceptable cost of inconsistent treatment of the age variable.

\section{Simulation Studies}

I will now use the basic equation 1 to generate data under various specifications for the parameters in this equation. The results obtained in these simulation studies show that it is quite possible to arrive at the correct con- 
clusions about the true sources of variation underlying the data, even in age-heterogeneous cross-sectional designs. In the simulation studies reported below, arbitrary numerical values have been assigned to parameters in the models used to generate data. No effort has been made to choose parameter values that would yield data that resemble, for instance, real human age distributions, because that would not affect the results and conclusions.

In the first simulation exercise, the extreme example presented in the paper based on equations $1.2-1.8$ will be used. This example uses a restricted version of equation 1:

$$
\mathrm{x}_{\mathrm{it}}=\left[\mathrm{t}_{\mathrm{i}}\right] \mathrm{S}_{\mathrm{x}}+\mathrm{e}_{\mathrm{xi}} \text { and } \mathrm{y}_{\mathrm{it}}=\left[\mathrm{t}_{\mathrm{i}}\right] \mathrm{S}_{\mathrm{y}}+\mathrm{e}_{\mathrm{yi}}
$$

In this restricted model, the $\mathrm{x}$ - and $\mathrm{y}$-scores of random subject $i$ only depend on this subject's age $\left[t_{i}\right]$, where the fixed regression coefficients are, respectively, $S_{x}$ and $S_{y}$. In addition, the additive error terms $\mathrm{e}_{\mathrm{xi}}$ and $\mathrm{e}_{\mathrm{yi}}$ are considered to be mutually uncorrelated. The authors show that even in this extreme case, the $\mathrm{x}$ - and $\mathrm{y}$-scores obeying this restricted model have nonzero correlation in the population of subjects. They then argue that this nonzero correlation '... should not be taken as evidence for a common causal aging mechanism or common process change within individuals over time'. Moreover, this nonzero correlation is called a 'spurious association between uncorrelated processes'. I do not agree with the statement that the nonzero correlation is not indicative of the presence of a 'common causal aging mechanism'. In the restricted version of equation 1 underlying the nonzero correlation, age $\left[\mathrm{t}_{\mathrm{i}}\right]$ is the explanatory variable common to both the $\mathrm{x}$ - and the $y$-scores. In contrast, the additional statement that the nonzero correlation is, for $\mathrm{x}$ - and $\mathrm{y}$-scores obeying the restricted model, not indicative of a common process change within individuals over time is correct. More specifically, this nonzero correlation is obtained even though there are no correlated random change components $\left[\mathrm{t}_{\mathrm{i}}\right] \mathrm{S}_{\mathrm{xi}}$ and $\left[\mathrm{t}_{\mathrm{i}}\right] \mathrm{S}_{\mathrm{yi}}$ in the restricted model.

In a nutshell, the main question to be addressed is whether a proper statistical analysis of data generated according to the restricted version of equation 1 indeed gives rise to the incorrect conclusion that the observed correlations are due to correlated random change components. I will present results from a simulation study which indicate that, even when considerable age heterogeneity is present in a cross-sectional setup, there is no danger of such a misinterpretation.

For 9,999 cases, scores have been generated on four variables according to the restricted version of equation 1 under consideration:

$$
\begin{aligned}
& \mathrm{y}_{1 \mathrm{it}}=\left[\mathrm{t}_{\mathrm{i}}\right] \cdot 0.8+\mathrm{e}_{1 \mathrm{yi}} \\
& \mathrm{y}_{2 \mathrm{it}}=\left[\mathrm{t}_{\mathrm{i}}\right] \cdot 0.7+\mathrm{e}_{2 \mathrm{yi}} \\
& \mathrm{y}_{3 \mathrm{it}}=\left[\mathrm{t}_{\mathrm{i}}\right] \cdot 0.6+\mathrm{e}_{3 \mathrm{yi}} \\
& \mathrm{y}_{4 \mathrm{it}}=\left[\mathrm{t}_{\mathrm{i}}\right] \cdot 0.5+\mathrm{e}_{4 \mathrm{yi}}
\end{aligned}
$$

In this simulation model, the error terms $\mathrm{e}_{\mathrm{jyi}}, \mathrm{j}=1,2,3,4$, are taken to be mutually uncorrelated standard normal variables. Also, age $\left[t_{i}\right]$ is taken to be a standard normal variable. The correlation matrix thus obtained is:

$\begin{array}{lllllll}\mathrm{y}_{1 \mathrm{t}} & 1.00 & & & & \\ \mathrm{y}_{2 \mathrm{t}} & 0.36 & 1.00 & & & \\ \mathrm{y}_{3 \mathrm{t}} & 0.32 & 0.30 & 1.00 & & \\ \mathrm{y}_{4 \mathrm{t}} & 0.28 & 0.26 & 0.23 & 1.00 & \\ {[\mathrm{t}]} & 0.62 & 0.58 & 0.52 & 0.45 & 1.00\end{array}$

If now age $\left[\mathrm{t}_{\mathrm{i}}\right]$ is partialled out, then the $(4,4)$-dimensional partial correlation matrix of the $y$-scores thus obtained is diagonal. This means that a multivariate regression of the four y-variables on age removes all the correlations. I carried out this and the following analyses by means of the Lisrel computer program [5]. Data simulation program and Lisrel input files can be obtained upon request. In conclusion, in the extreme case considered by the authors, there is no danger of misinterpretation of the observed correlations, if the proper statistical analysis is carried out. That is, if age is partialled out, then all observed correlations are removed, and hence there is no need anymore to consider correlated random change components.

In the second simulation experiment, I will use the full basic equation 1 to generate data. For 9,999 cases, scores have been generated on four variables according to the following instance of equation 1 :

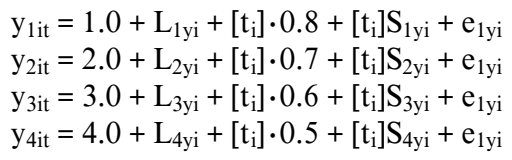

In this simulation model, the error terms $e_{j y i}, j=1,2,3,4$, are again taken to be mutually uncorrelated standard normal variables. The random intercepts $\mathrm{L}_{\mathrm{jyi}}, \mathrm{j}=1,2,3,4$, and the random regression coefficients $\mathrm{S}_{\mathrm{jyi}}, \mathrm{j}=1,2,3,4$, also are taken to be mutually uncorrelated standard normal variables. The age $\left[\mathrm{t}_{\mathrm{i}}\right]$ is taken to be normally distributed with a mean of 5.0 and a variance of 25.0. The correlation matrix thus obtained is:

$$
\begin{array}{llllll}
\mathrm{y}_{1 \mathrm{t}} & 1.00 & & & & \\
\mathrm{y}_{2 \mathrm{t}} & 0.21 & 1.00 & & & \\
\mathrm{y}_{3 \mathrm{t}} & 0.19 & 0.17 & 1.00 & & \\
\mathrm{y}_{4 \mathrm{t}} & 0.17 & 0.14 & 0.13 & 1.00 & \\
{[\mathrm{t}]} & 0.50 & 0.44 & 0.38 & 0.35 & 1.00
\end{array}
$$


The obtained mean values are:

$$
\begin{array}{lllll}
\mathrm{y}_{1 \mathrm{t}} & \mathrm{y}_{2 \mathrm{t}} & \mathrm{y}_{3 \mathrm{t}} & \mathrm{y}_{4 \mathrm{t}} & {[\mathrm{t}]} \\
5.07 & 5.57 & 5.91 & 6.52 & 5.02
\end{array}
$$

If a multivariate regression is carried out of the four $y$-variables on age, then this again removes all correlations between the y-variables. That is, the partial correlation matrix of the four $y$-scores after regressing out age is diagonal. The conclusion, therefore, is again that also for the full basic model 1 there is no danger of misinterpretation of the observed correlations in these age-heterogeneous cross-sectional data, if age is partialled out. The diagonal partial correlation matrix thus obtained precludes the need to consider correlated random change components.

In the final simulation study, again the full basic equation 1 is used. The parameter values in the simulation model are exactly the same as in the previous (second) simulation run. The only difference is that now the random change components $S_{\mathrm{jyi}}, \mathrm{j}=1,2,3,4$, are taken to be correlated. That is, $\operatorname{cor}\left[\mathrm{S}_{\mathrm{jyi}}, \mathrm{S}_{\mathrm{kyi}}\right]=0.5, \mathrm{j} \neq \mathrm{k} ; \mathrm{j}, \mathrm{k}=1,2,3$, 4. The correlation matrix now obtained is:

$$
\begin{array}{llllll}
\mathrm{y}_{1 \mathrm{t}} & 1.00 & & & & \\
\mathrm{y}_{2 \mathrm{t}} & 0.59 & 1.00 & & & \\
\mathrm{y}_{3 \mathrm{t}} & 0.56 & 0.55 & 1.00 & & \\
\mathrm{y}_{4 \mathrm{t}} & 0.53 & 0.53 & 0.54 & 1.00 & \\
{[\mathrm{t}]} & 0.49 & 0.41 & 0.37 & 0.31 & 1.00
\end{array}
$$

The obtained mean values are:

$\begin{array}{lllll}\mathrm{y}_{1 \mathrm{t}} & \mathrm{y}_{2 \mathrm{t}} & \mathrm{y}_{3 \mathrm{t}} & \mathrm{y}_{4 \mathrm{t}} & {[\mathrm{t}]} \\ 5.02 & 5.46 & 6.03 & 6.43 & 5.02\end{array}$

If now multivariate regression is carried out of the four $y$-variables on age, then the $(4,4)$-dimensional correlation matrix of the y-scores thus obtained is not longer diagonal. This partial correlation matrix after regression out age now is:

$\begin{array}{lllll}\mathrm{y}_{1 \mathrm{t}} & 1.00 & & & \\ \mathrm{y}_{2 \mathrm{t}} & 0.49 & 1.00 & & \\ \mathrm{y}_{3 \mathrm{t}} & 0.47 & 0.47 & 1.00 & \\ \mathrm{y}_{4 \mathrm{t}} & 0.46 & 0.47 & 0.48 & 1.00\end{array}$

Clearly, this partial correlation matrix now will have to be explained by either correlated random intercepts $L_{j y i}, j=$ $1,2,3,4$, or correlated random regression coefficients $\mathrm{S}_{\mathrm{jyi}}$, $\mathrm{j}=1,2,3,4$. Without further constraints this yields a saturated model for standard cross-sectional data.

I conclude from these simulation studies that there is no danger of misinterpretation of observed correlations in age-heterogeneous cross-sectional studies. As is common practice in structural equation modeling, one fits several alternative models to the observed correlation matrices and means. Among these alternative models are ones in which age is partialled out. If the partial correlation matrix thus obtained is diagonal, then there is no need to invoke correlated age-dependent change processes. However, if partialling out age does not remove all correlations, then models involving correlated change processes or correlated random intercepts can be considered.

Given the importance of also considering models for age-heterogeneous cross-sectional data in which age is partialled out, as shown by the simulation studies, I do not understand the critical remarks made by the authors about regressing out age. For instance they state that '... partialling for chronological age variance only partially removes true age-based effects that results from covariance associated with the cumulative effects of correlated rates of change'. This is not entirely correct, as shown by my third simulation study. Partialling out age does not at all remove the correlation between the random rates of change $S_{\mathrm{jyi}}$ [see the $(4,4)$-dimensional partial correlation matrix of the $y$-scores given above]. This is not a vice, but a virtue, because it makes it possible in the next modeling step to introduce the random change components $\left[\mathrm{t}_{\mathrm{i}}\right] \mathrm{S}_{\mathrm{jyi}}$ in order to explain these partial correlations.

\section{Longitudinal Designs}

The authors indicate that longitudinal designs provide the best information concerning developmental processes. I think that their point of view is shared by most social and behavioral scientists. Yet I would like to take this opportunity and qualify the kind of information that can be obtained in all designs and statistical analyses in which pooling across subjects occurs. These designs include cross-sectional, sequential, and longitudinal setups, as well as hybrid combinations thereof. In those approaches, the unit of analysis is between-subject variation. Even in longitudinal analyses, the longitudinal covariance matrix and longitudinal mean vector is computed by pooling across subjects. This necessarily implies the strong assumption that subjects are homogeneous in all relevant aspects - they are interchangeable with other subjects in the population under consideration. Even in hierarchical modeling, it has to be assumed that all subjects obey the same model structure (e.g., linear growth) and that the random coefficients characterizing individual subjects (like $S_{\text {jyi }}$ in equation 1) have the same distribu- 
tion across subjects. Furthermore, importantly, it is assumed that such analyses of between-subject variation in various forms provide reliable information of the structure of within-subject variation of each subject in the population.

In collaboration with Nesselroade (University of Virginia), we have shown that analyses of between-subject variation (like in cross-sectional, sequential, and longitudinal designs) in most cases (namely those cases where age-dependent changes occur, and that is of course the focal interest of developmental investigations) do not at all provide reliable information about the structure of intraindividual variation (i.e., each individual ontogenetic trajectory) [6]. In fact, this can be proved by means of mathematical-statistical techniques, but we also showed it in a number of simulation studies and applications to real data. Equally disturbing is our finding (again by means of mathematical proof as well as large-scale simulation experiments) that in factor analyses of between-subject variation (i.e., the typical approaches in cross-sectional, sequential, and longitudinal studies) the presence of substantial between-subject heterogeneity in fixed model parameters (like factor loadings) remains entirely invisible. Hence one can obtain a satisfactorily fitting longitudinal factor model, while the true within-subject factor structure of each subject in the longitudinal sample is completely different, and none of the within-subject factor loadings equals the loadings obtained in the longitudinal analysis. These results imply that the foundations of psychometrics, in particular test theory, require major extensions. For instance, results obtained in analyses of between-subject variation are in most cases entirely unreliable when applied in individual assessment and counseling.

This is not the proper place to further elaborate these new methodological developments. Suffice it to say that multivariate time series techniques (like Cattell's P technique) obtain a new and central role in this extended psychometrics and test theory. I refer to Molenaar et al. [6] for further information.

\section{General Discussion and Conclusions}

The authors regard correlations which are due to mean age trends (described by the term $\left[\mathrm{t}_{\mathrm{i}}\right] \mathrm{S}_{\mathrm{x}}$ in their basic model) as spurious correlations. I do not agree with this point of view. Mean age trends also require explanation, because they are an important parameter of the complete agedependent distributions. I agree with the point of view of Wohlwill [3] regarding the possible explanatory role of age, but this is quite compatible with the point of view that mean age changes also require explanation. These mean age changes are not spurious, only their possible explanations could be spurious. The same can be concluded with regard to random age-dependent changes and their mutual correlations (like the $\left[\mathrm{t}_{\mathrm{i}}\right] \mathrm{S}_{\mathrm{jyi}}$ terms in the basic equation). These correlations also are not spurious, but require explanation (where only their explanation could be spurious).

The authors correctly indicate that the use of narrow age cohort designs controls for age heterogeneity. Indeed, fixing all $\left[\mathrm{t}_{\mathrm{i}}\right]$ terms at the same value, $\left[\mathrm{t}_{\mathrm{i}}\right]=\mathrm{c}$, only leaves open the possibility that the residual $\mathrm{e}_{\mathrm{jyi}}$ terms in their basic model are correlated. And of course this also requires explanation, although I doubt that such explanations could be regarded as developmental explanations. Using sequential narrow age cohort designs then can provide information about the short-term covariation of these residual terms (which could be interpreted as statelike variables). To obtain interesting information concerning developmental processes, multigroup designs employing several distinct narrow age groups would seem to be more interesting. The same remarks apply to the use of sequential variants of such narrow age group designs; they can provide useful short-term information concerning state-like developmental processes.

My main message to the authors is that all these different forms of information (and like I indicated in the previous section, also additional forms of intra-individual time series information) are potentially equally interesting and require causal explanation. It is up to researchers to decide which type of information they consider interesting in each particular situation.

\section{References}

1 Hofer SM, Sliwinski MJ: Understanding ageing: An evaluation of research designs for assessing the interdependence of ageing-related changes. Gerontology 2001:47:341-352.

2 Ellis JL, Junker B: Tail-measurability in monotone latent variable models. Psychometrika 1997;62:495-523

3 Wohlwill JF: The Study of Behavioral Development. New York, Academic Press, 1973.
4 Molenaar PCM, Boomsma DI, Neeleman D, Dolan CV: Using factor scores to detect $\mathrm{G} \times \mathrm{E}$ interactive origin of 'pure' genetic or environmental factors obtained in genetic covariance structure analysis. Genet Epidemiol 1990;7: 93-100.
5 Jöreskog KG, Sörbom D: Lisrel ${ }^{\circledR}$ 8. Chicago, Scientific Software International, 1993.

6 Molenaar PCM, Huizenga HM, Nesselroade JR: The relationship between the structure of interindividual and intra-individual variability: A theoretical and empirical vindication of developmental systems theory; in Staudinger UM, Lindenberger U (eds): Understanding Human Development. Dordrecht, Kluwer, in press. 